\title{
3D COLOUR SCANS FOR OBJECT ASSESSMENT
}

Mona Hess (UCL Museums and Collections), Francesca Simon Millar (Anthropology, UCL), Yean-Hoon Ong (UCL Museums and Collections),

Stuart Robson (Civil, Environmental and Geomatic Engineering, UCL), Graeme Were (Anthropology, UCL), Ian Brown (Computer Science, Oxford University), Sally

MacDonald (UCL Museums and Collections).

UCL Museums and Collections

University College London

Gower Street

London WC1E 6BT

United Kingdom

m.hess@ucl.ac.uk, f.millar@ucl.ac.uk, yean-hoon.ong@ucl.ac.uk, srobson@cege.ucl.ac.uk, g.were@ucl.ac.uk, ian.brown@oii.ox.ac.uk, s.macdonald@ucl.ac.uk, e-curator@ucl.ac.uk

URL: http://www.ucl.ac.uk/museums/research/ecurator

Abstract - Digital heritage technologies are radically changing the way we engage with material culture and are negotiating new ways of knowing and understanding the object. Realising the importance of digital technologies and new interdisciplinary possibilities, the E-curator project has been undertaken by UCL Museums and Collections with the goal of applying two state of the art digital technologies: 3D colour laser scanning and e-Science technologies. This project captures and shares very large three-dimensional scans and detailed datasets about museum artefacts in a secure computing environment. This paper presents our investigation about the use of 3D colour scanning for museum object assessment.

\section{INTRODUCTION}

The E-curator project currently in progress at University College London (UCL), Department of Museums and Collections, is an interdisciplinary project which draws on UCL's expertise both in curatorship and in e-Science. It is a one-year project which aims to (a) develop a traceable methodology for recording the surface detail and colour quality of a range of object types and materials. (b) Explore the potential for producing validated datasets that would allow closer and more scientific examination of groups of objects, the processes involved in their manufacture, and issues of wear and deterioration. (c) Examine how the resulting datasets could be transmitted, shared and compared between disparate locations and institutions. (d) To begin to build expertise in the use and transmission of 3D scan data as a curatorial tool.

The E-curator project is an opportunity to exploit and test e-science technologies and explore some of the opportunities they offer museum practice in a virtual world. Such deployment will enable curators and conservators to compare records collected at different institutions stored remotely, or collected over a period of time under different conditions, in order to assess and monitor change. Furthermore it provides an 
opportunity to test close-range scanning methods and critically assess their capability for heritage recording and documentation.

Six museum objects have been selected from UCL Museums and Collections to form the basic study of the E-curator project. Each object has curatorial or conservational questions embedded in its form that 3D colour digitisation combined with a digital curatorial process seeks to capture and present to the museum user. Each of these objects has been scanned using a state of the art Arius3D Foundation Model 150 colour scanner, which is housed at UCL and is the highest resolution and most geometrically accurate 3D colour laser scanning system currently in the UK. The 3D object scans and relevant catalogue information are stored on an e-Science storage system, Storage Resource Broker (SRB). Curators and conservators can remotely access the 3D object scans and catalogue information via the E-curator website.

\section{CONVENTIONAL TECHNIQUES}

Whilst the direct encounter with the material object will always have value for conservators and curators and includes an important learning process, museum specialists have traditionally used a combination of photography and text to prepare illustrated catalogues and reports for the purposes of object identification, objecttracking, object comparison and condition checking. Such examinations are typically made within an institution and when objects travel in touring exhibitions. Conventional imaging techniques have always been strictly two-dimensional, be it drawings (even axonometric projections), photographs, or 2D scans of a document. These standard record formats have the limitation of being very selective and insufficient to record nuanced information about the complete shape, colour and texture of an object.

The highest standards of documentation of artefacts, as required by the 'Code of Ethics' for Museums [1] and Conservators, will change in future and limitations will become clear with new methods arising. Today some of the limitations of conventional documentation techniques can be addressed by using 3D colour scanning, which has the ability to record the whole object in the round and in great detail and by disseminating the data with a shared web-based database platform. These methods should now and in future become an accessible tool for heritage specialists.

\section{D COLOUR LASER SCANNING}

The application of Virtual Heritage [2] in art history, conservation and curation with developments of digital tools and new interfaces is a relatively new phenomenon within the 200 year old tradition of European heritage conservation and preservation.

To advance manufacturing capabilities the engineering sector has developed precise measurement tools and more recently best practice guidelines linked to internationally accredited standards. Possibly the most relevant of these technologies for heritage recording is $3 \mathrm{D}$ colour laser scanning which has been in development since the early 1990s [3]. Established understanding of these sensing technologies provides an established body of knowledge that can be directly exploited by the heritage sector to foster the rapid adoption of digital recording techniques.

\section{Technical equipment}


The 'Arius3D Foundation Model 150' scanner $^{1}$ offers a detailed non-contact and non-destructive documentation and examination method which predetermines its use for conservation recording.

Surface scanning is carried out by a scanner head which emits three laser beams of different wavelengths, red (R), green ( $\mathrm{g}$ ) and blue (B), in a focused white laser beam. The scan head simultaneously measures colour reflectance and geometry by triangulation between the laser and a camera, recording the laser reflection at each location illuminated by the lasers. Every point has therefore a XYZ coordinate location and an RGB colour value. A calibrated 'white cube' is used to supply data to enable any variations in background illumination and laser output power to be corrected on a scan line by scan line basis as part of an off-line colour calibration process. The Arius3D scanner head is mounted to a motion control system (Coordinate Measuring Machine), which moves it over the object. The single point accuracy of the scanning head in use at UCL is better than 25 microns in depth with the scan head and motion control system delivering a minimum spatial sampling interval of 100 microns. This sampling interval is commensurate with the laser spot diameter used to sample the surface within the field of view of the scanning head. To ensure consistent dimensional capability the unit is installed in an air-conditioned room that maintains temperature at 20 degrees and can control relative humidity to suit the objects being recorded.

\section{The $3 \mathrm{~d}$ colour laser scanning process}

Our objective for the scanning part of the project is to document the artefacts very precisely in geometry and colour to produce a virtual three dimensional archival document that widens our knowledge of the object. The scan product is thus a highfidelity coloured three-dimensional point cloud with a point spacing of $100 \mu \mathrm{m}$. Such a detailed survey has not been delivered before previously for any of the objects selected for the core study.

As an illustrative case study this paper follows the process of scanning and browsing one of the core objects from UCL Museums and Collections in the E-curator application. The chosen example is a medicine bundle (collection number M99) from Nigeria, housed at the UCL Ethnographic Collection (Figure 1). It is described as 'container of twisted copper wire on outside, inside is a piece of ant-hill to be used as medicine and to be worn at waist', a 'carrying device' and a 'ritual object', the materials being clay and metal. ${ }^{2}$ The small object is about $8 \times 8 \times 3 \mathrm{~cm}$. It is a piece of earth or anthill bound with a length of wire used as a charm or for its curative powers. A U-shaped handle, made of the same wire, fixes the wire to the earthen body. The wire is badly corroding in places. A 3D scan is hoped to reveal greater surface detail of the wire and thus provides a holistic analysis of surface corrosion for continual monitoring rather than a record of selected points of extreme corrosion. The object is extremely fragile and cannot be positioned on some of its sides because of the weak flexibility of the wire frame (Figure 1). It was chosen as part of the study because of its fragility making it a conservation issue and for its mixed composition of materials thus pushing the boundaries of the capabilities of the Arius3D scanner.

\footnotetext{
${ }^{1}$ Arius 3D Inc., Ontario, Canada, www.arius3d.com accessed 28 May 2008.

${ }^{2}$ Collector Shute, D. A. F. ; UCL Ethnographic Collection Filemaker database, accessed 18 May 2008.
} 
The geometry of the medicine bundle is complex with many occlusions of the earthen centre by the wire container which present significant challenges for the optical method of laser scanning. The back of the wires touching the anthill and the surface of the anthill covered by wire could not be recorded. In general the surfaces reflected sufficient laser illumination and scanned very well. The object was placed on a turntable so that it could be rotated to provide optimal scanning viewpoints whilst reducing the physical contact with the object during the scanning process as much as possible. For recording the maximum amount of geometry in different positions it was sometimes necessary to support and stabilize the medicine bundle with conservator approved acid free pencil erasers. The object was then scanned in the same position from as many precalibrated scanning head angles as possible. A total of 8 scan data files each containing several individual scanning records were produced.

In this case of this complex object the scanning process took one working day whereas the post-processing took two to three days. The work on the recorded data consists of several consecutive steps. First the raw scan data is imported into the proprietary Arius3D, Pointstream 3D Image Suite software ${ }^{3}$, which is specifically designed software to process and visualize the densely sampled 'point cloud' data. The next step controls the optimum alignment of the different component scan records to best fit using a point cloud to point cloud iterative closest point procedure. Then the point clouds are cleaned to remove overlapping geometry using both manual editing and automatic filtering of multiple points based on combinations of geometry and colour content. After editing the geometry and colour information the component records are merged to a single scan image.

The final medicine bundle data set consists of a total of 41 scan records held within 8 scan files each of which represents an positions on the turn table used to rotate the object with minimal manual handling. The file consists of 1.8 million 3D colour points at 100 microns spacing, the complete binary data file size is 30MB. Further processing was then employed to fill any occlusions in the data set with point grids. These additional addenda interpolate both geometry and colour data and serve to complete the picture for educational reasons, files structures are arranged so that the user can distinguish between areas of measured and interpolated information.

An Active-X Pointstream software display plug-in provides a viewing method within the E-curator interface so that users can conveniently visualise and interact with the coloured point cloud. The dense 3D point cloud image representation eliminates the reverse engineering process of fitting polygons to point cloud data. Thus the geometric information does not require any triangulation process which keeps data file sizes to a minimum and avoids a significant additional modelling and interpolation step which is more usual for scanning systems delivering relatively spares data sets by comparison.

\section{Impact of 3D documentation on conservation and curatorship}

Viewing and handling of the real object provides an essential comparison against which to assess the value of real time user interaction with the 3D digital colour model within the E-curator user interface by museum specialists. By these comparative means the digital image can be explored as a curatorial tool as it, for example, allows the eye to

\footnotetext{
${ }^{3}$ Pointstream 3D Image Suite Software (Version 2.9.0.0.) by Pointstream Inc., Canada, www.pointstream.net accessed 28 May 2008
} 
read the surface of an object, navigating inscriptions, paint strokes and the topology of the object. We are currently investigating how conservators can use the capabilities of the detailed and minute 3D documentation process to manage, understand and conserve the real object. The 3D documentation process can also draw on the dimensional and colour fidelity of a temporal succession of such digital records to allow reliable comparison and monitoring of the object over time. Visualisation of the 3D digital record allows control of both lighting situation and surface specularity in order to highlight geometric features in ways which parallel traditional raking light analysis used to find hidden brushwork, forms or structure. Cross sections of objects can be produced, directly from the dense point cloud data, to provide a more traditional representation to assist in the evaluation of change and to support the analytical analysis of object form and geometry as a teaching aid in conservation. As an example, to enhance use in teaching and learning, the components of the medicine bundle, the earthen ant hill and the copper wire, have been separated into different layers allowing students to study the form and composition of different elements of the artefact. The 3D models also allow curators to create virtual exhibition display scenes. Further dissemination methods are made available through the final data set of the 3D record of an object: virtual reconstructions, animations and films for multimedia and education. Once scanning capture and data processing best practice are established, the method will provide an invaluable tool to test authenticity, to confirm authorship and to assess any change of damage following a loan by comparison of 3D data surface information.

\section{User designed interface}

The novel approach and a particular strength of the E-curator project is in the expressed participation of all stakeholders into the design and development process at all stages. These range from the initial condition report through the scanning, data processing and visualisation of the selected objects to the final use of the application.

During the project, a number of internal and external formative workshops are being held to offer curators, conservators and other museum specialists at UCL the opportunity to look in more detail at the proposed project and produce a more detailed specification and review criteria. These workshops are also allowing the team to refine the user interface of the E-curator application, and to understand curatorial requirements for labelling and linking to external data sources.

The first workshop took place in February 2008 at UCL. Several curators and conservators from UCL Museums were invited by the steering group. The goal of the workshop was to find out what capabilities the users would like have within the Ecurator application and to seek an optimal outcome for designing the interface for the web-based software tool. By consulting and involving cultural heritage specialists from the commencement of the project we will find out what they require from the 3D image and be able to develop an interface to serve as a useful tool. A participatory approach to user designed systems was used during the workshop. It began with a 'Condition Report and Catalogue Entry' session, in which curators and conservators demonstrated the preparation of condition report and catalogue entry using objects from UCL Museums and Collections. A brainstorming session was then carried out to tease out common requirements from the broad range of specialisms represented by those attending. Participants were requested to write a list of the features they would think as being most useful within the software. These features were then brought together and were ranked 
according to their priorities. A second workshop to be held during the summer will have a similar approach.

During the second half of the project duration single users will be asked to browse their objects on a prototype of the E-curator application and the direct one-to-one feedback will influence the programming. Evaluation sessions with curators and conservators and a summative workshop contribute will round up the software development phase.

\section{D COLOUR SCANS FOR OBJECT ASSESSMENT}

The use of digital technologies within the museum environment has encouraged the development of various software applications, which are used to manage the text and image records of museum artefacts. Most of the images administered by these museum applications are 2D images. In our E-curator project, a web application is developed to manage 3D images and relevant metadata.

For 3D colour scans to be of practical use, robust means of sharing and validating the data obtained need to be established. High resolution colour scans of one object can require hundreds of megabytes of storage space, and can only realistically be shared using the distributed file systems such as Storage Resource Broker (SRB) ${ }^{4}$ being widely deployed in the e-Science environment.

SRB is a data grid middleware software system produced by the San Diego Supercomputer Centre (SDSC). The system implements a logical namespaces (distinct from physical file names) and maintains metadata on data-objects (files), users, groups, resources, collections, and other items in an SRB Metadata Catalogue (MCAT) stored in a relational database management system. System and user-defined metadata can be queried to locate files based on attributes as well as name. The SRB system is middleware in the sense that it is built on top of other major software packages (various storage systems, real-time data sources, a relational database management system etc) and it has callable library functions that can be utilized by higher level software. However, it is more complete than many middleware software systems as it implements a comprehensive distributed data management environment, including various end-user client applications. It has features to support the management and collaborative (and controlled) sharing, publication, replication, transfer, and preservation of distributed data collections.

3D scans in Arius3D Pointstream format are loaded into SRB server using the SRB client software called inQ ${ }^{5}$ (Figure 2). These images are organized as a collection hierarchy. Images from different museums, for example, can be stored in separate subcollections.

The metadata used to describe the 3D images generated within E-curator is based on SPECTRUM $^{6}$, the UK Museum Documentation Standard, for catalogue entries. These metadata provides information about the object ID, physical description, location, historical facts, condition, exhibition and conservation information.

A web-based interface is created for accessing the 3D scan collection by users consisting of museum specialists such as curators and conservators. The interface provides both browsing support for examining the collection hierarchy, and analysis

\footnotetext{
${ }^{4}$ http://www.sdsc.edu/srb/index.php/Main_Page, accessed on 18 May 2008.

${ }^{5} \mathrm{http}: / / \mathrm{www} . \mathrm{sdsc} . \mathrm{edu} / \mathrm{srb} / \mathrm{index} . \mathrm{php} / \mathrm{InQ}$, accessed on 18 May 2008.

${ }^{6} \mathrm{http}: / / \mathrm{www} . \mathrm{mda}$.org.uk/spectrum.htm, accessed on 18 May 2008.
} 
tools for manipulating both 3D and conventional 2D images. When the user clicks on an object, the 3D scan is downloaded from SRB to web browser through the web server. To view the 3D coloured point cloud, users need to install an ActiveX control from Arius3D called 3DImageViewer. For each artefact, tools are available to allow users to alter for example lighting conditions or colour appearance and to view cross sections and check key dimensions of the objects. The web interface also allows users to display multiple 3D images at the same time and thus facilitates the visual comparison of collections of museums artefacts.

The functionalities supported by the E-curator website can be illustrated through our exemplar scenario. When users access the E-curator application, a list of 3D scan images from different collections will be displayed (Figure 3). Consider a user who wants to view and analyse the medicine bundle from the anthropology collection. When the user clicks on the object, a new window (Figure ) pops up displaying the 3D image and relevant metadata of the medicine bundle. By clicking on the small icons at the bottom of the 3D image, the user can tumble, pan, zoom and rotate the 3D image to explore the surface of the medicine bundle. The user can also toggle the lighting to examine the geometric variations on the surface. Links to different sets of raw and processed data are provided if the user is interested in examining the 3D images generated at different scanning and data processing stages. A hierarchy for these records has been established including the aligned 'registered' version of the point cloud without colour or point processing, a 'processed' version with cleaned colours and geometry, and a 'presentation' file with optimised colours and filled data voids. For the use of conservators and curators the second model will be the most relevant since it encompasses the complete object in one data set but has undergone the least data alteration and processing.

Thus the website and its SRB supporting system is designed to function as an essential interface for object identification and assessment. With an appropriate storage infrastructure, data sets may also be interrogated more widely by researchers around the world.

\section{Object Metadata}

User access to metadata within the E-curator application allows further study of the history, exhibition and conservation information of each of the objects.

3D representations of heritage objects and museum artefacts need to have a clear 'provenance'. On the one hand a description of history and ownership of the object, on the other hand a clear set of data that proves the genesis of the digital 3D files of the object. Bearing this in mind we developed a specific metadata set for the E-curator application that includes UCL Museums and Collections catalogue entries, conservation reports, images and photographs, microscopic images. Every three-dimensional record is also annotated with metadata concerning its capture, the import and colour calibration filters used, and any particular scan process and post processing information. This dataset provides the user with clear information about the production and the authorship of the 3D image. The development of the metadata set has largely followed the recommendations of English Heritage for Scandata [4] and 'Big Data' project [5] for Arts and Humanities data.

The Arius3D Scanner offers unique and new possibilities to fulfill the claim of the Codes of Ethics of heritage professionals to use the tools for best documentation if 
traditional techniques cannot record all features. The German VDI/VDE 2634 guidelines give technical guidelines for the calibration and the measurements used in optical 3D measuring systems and aims to control the vocabulary [6]. But traceable procedures and standards for good practice for close range 3D laser documentation must be further developed [7] to make it a useful tool in the heritage sector. Dissemination and archiving must be further developed. Even though the 'Big Data Project' recommends .LAS, a LIDAR (airborne laser scanning) scanning standard, [5, p37] however a common file format for archiving 3D scans in the museum sector is yet to be agreed on and must yet be formalised.

The current version of the London Charter "For the use of 3-dimensional visualisation in the research and communication of cultural heritage" [8 \& 9] is directed towards some of the problems and possibilities that arise from 3D colour scanning as a documentation tool in heritage documentation and thus to the E-curator research project, but it does not yet embrace established engineering metrology approaches which could provide a common basis for 3D data comparison. Our project tries to approach the process of the 3D scanning and visualisation work with 'intellectual and technical rigour' and tries to create a 'methodological approach' to 3D colour laser scanning. The careful creation of metadata should facilitate the 'Transparency Requirements' (Principle 4) of the process and we aim to fulfill the 'documentation of the interpretative decisions made in the course of a 3D visualisation' (Section 4.5). The London Charter has direct influence on the 'metadata and paradata' which are developed in the E-curator project and results of the project will need to be discussed with a subject community to develop 'appropriate standards and ontologies', e.g. 3D Laser Scanning for Museums as suggested in Section 6.1.

e-Science is developing new tools and methods. The E-curator project aims to find new solutions for museums and heritage specialists.

\section{CONCLUSION}

E-curator, with its basis on the proven Storage Resource Broker (SRB) system and Arius 3D colour scanning technology has brought e-Science and 3D colour recording technologies together to develop a web based access system for 3D colour digital records of museum and cultural objects. The processes and procedures adopted follow and further develop best practice in disciplines ranging from museum curatorship, engineering metrology, computer science and cultural object handling to deliver a state of the art e-Science application which is being proven by experts in all three fields.

The user designed interface takes into account the everyday practices of conservators and curators in an encounter with the real. This encounter is replicated in the animation of the digital copy as tools allow the user to inspect the surface and carry out preliminary observations. Presently the project is restricted to a few objects and the tools that are incorporated at present do not compete with an interanimated tactile relationship with the real. However they do offer a reconfiguration of practice and suggest a step forwards at a virtual engagement with objects. In this realm of Virtual Heritage, examination of the surface materiality and object topology is possible through detailed 3D documentation. It is useful to further note the possibilities that can be built on the E-curator project incorporating haptic technology and microscopic images into the $3 \mathrm{D}$ structure. 
The strength of the E-curator project is the expressed participation of all stakeholders that will 'use' the application into the design and development process. Thus the website will function as an essential interface for object assessment which can be built upon to include a wider selection of objects and a richer tool repertoire.

The developed system, a novel cultural heritage application, accessed through its supporting website, has the capability to provide an interface and storage infrastructure able to support the dissemination of both existing museum records and a hierarchy of 3D colour models to a global audience.FIGURES

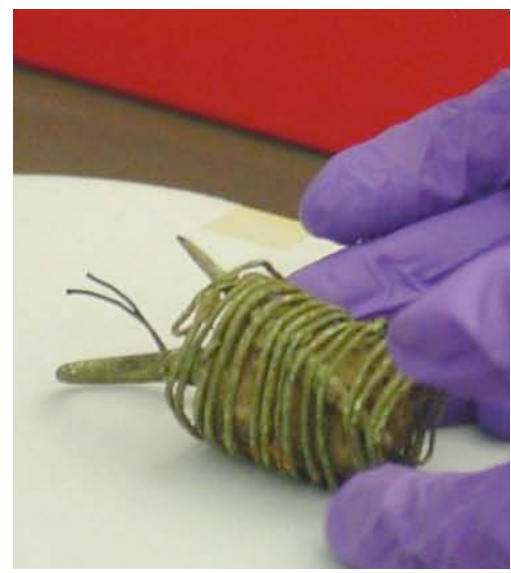

Figure 1. The medicine bundle, a core object in the E-curator study, is placed on the turntable by the conservator for scanning.

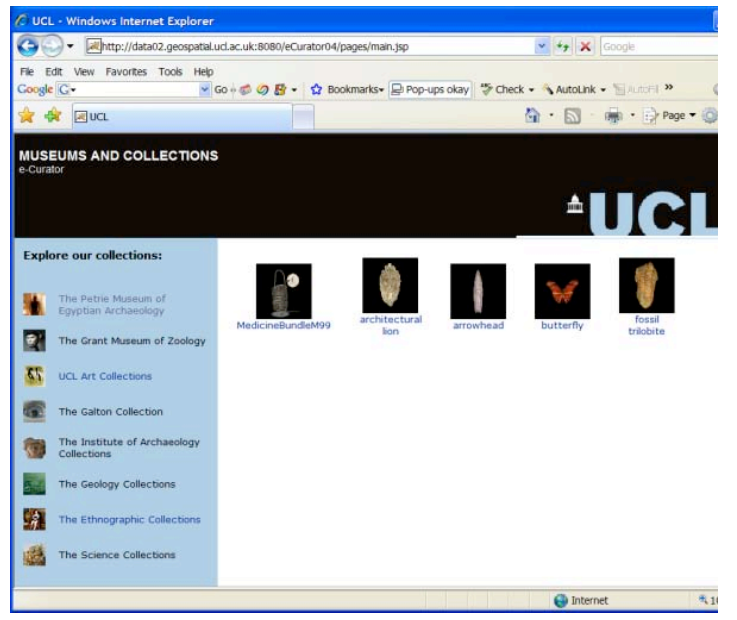

Figure 3. E-curator main page showing the $3 \mathrm{D}$ scan collections

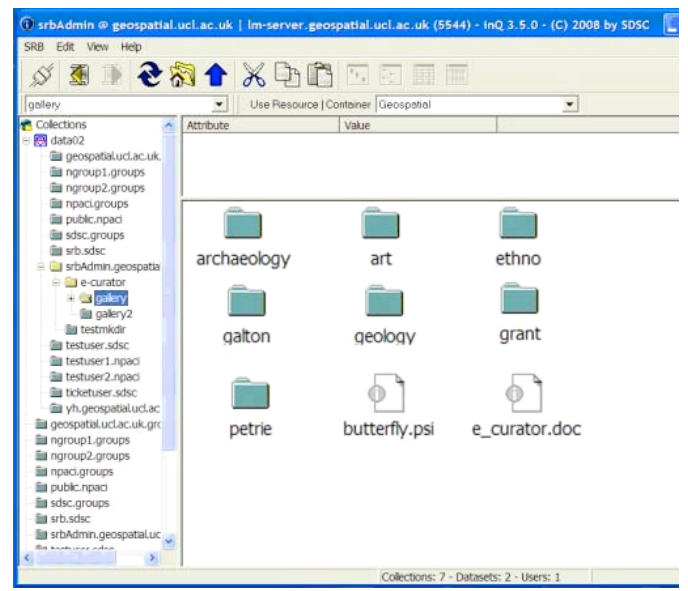

Figure 2. inQ interface (SRB client software)

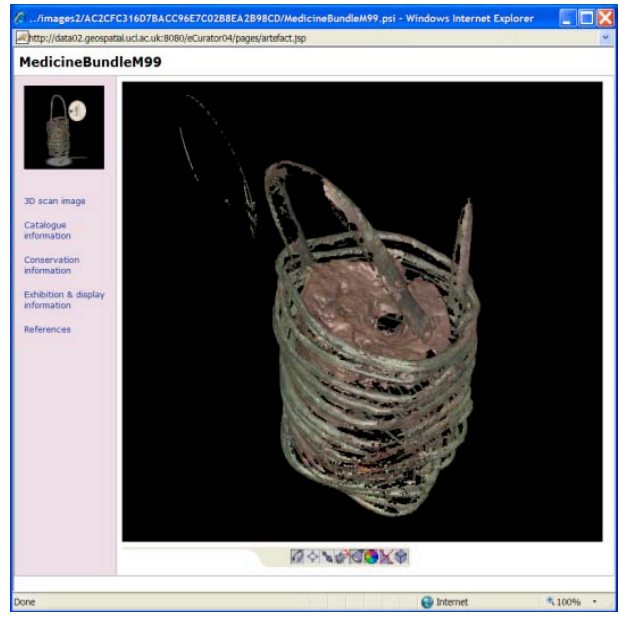

Figure 4. The medicine bundle in the E-curator application browser window. Metadata and information can be opened by clicking on the left bar. 


\section{ACKNOWLEDGEMENTS}

The project is jointly funded by the Arts and Humanities Research Council (AHRC), the Engineering and Physical Sciences Research Council (EPSRC) and the Joint Information Systems Committee (JISC).

We would like to thank the participants of the E-curator workshop at UCL in February 2008 for their support and feedback: Dr Andrea Fredericksen (UCL Art Collection), Sarah Mengler (UCL Ethnographic Collection), Susanna Pancaldo (UCL Petrie Museum), Elizabeth M Pye (UCL Archaeology), Dr Stephen G J Quirke (UCL Petrie Museum), E S (Libby) Sheldon (UCL History of Art), Kalliopi Vacharopoulou (UCL Museums and Collections),.

\section{References}

[1] ICOM: Statues and Code of Professional Ethics International Council for Museums - ICOM 1996). URL: http://icom.museum/ethics.html\#intro , accessed 11 May 2008.

[2] ROUSSOU, M.: The components of Engagement in Virtual Heritage Environments. in New Heritage. New Media and Cultural Heritage. Kalay, Y. E. , Kvan, T. , Afflek, J. (editors), New York, 2008.

[3] BARIBEAU, R; GODIN, G; COURNOYER, L and RIOUX, M: Colour ThreeDimensional Modelling of Museum Objects. In HIGGINS, T et al (editors), Imaging the Past. Electronic Imaging and Computer Graphics in Museums and Archaeology. British Museum, Occasional Paper, Number 114. 1996.

[4] ENGLISH HERITAGE: An Addendum to the Metric Survey Specifications for English Heritage. Addendum 2: A standard specification for the collection of point cloud data by terrestrial laser scanning or other methods.

[5] AUSTIN, T and MITCHAM, J; ARCHAEOLOGY DATA SERVICE and ENGLISH HERITAGE: Preservation and Management Strategies for Exceptionally Large Data Formats: 'Big Data' (EH Project No. 3948), Final Version 1.03, 28. September 2007. URL: http://ads.ahds.ac.uk/project/bigdata/ accessed on 18 May 2008.

[6] VERBAND DEUTSCHER INGENIEURE - VDI/VDE-Gesellschaft Mess- und Automatisierungstechnich (GMA): German VDI/VDE Guidelines 2634 - part 3. Optical 3D-measuring systems - Multiple view systems based on area scanning. September 2006. Beuth Verlag, Berlin. (This third part is still to be published in English.)

[7] BERALDIN, J.-A., National Research Council Canada: Digital 3D Imaging and Modelling: A Metrological Approach. 2008 in Time Compression Technologies Magazine, January/February 2008, pp.35-35, NRC 49887

[8] THE LONDON CHARTER INTEREST GROUP: The London Charter. For the use of 3-dimensional visualisation in the research and communication of cultural heritage. Draft 1.1 - 14 June 2006. URL: www.londoncharter.org accessed on 18 May 2008.

[9] BEACHAML, $\mathrm{R}$ and DENARD, $\mathrm{H}$ and NICCOLUCCI, F: An Introduction to the London Charter. In The E-volution of ICTechnology in Cultural Heritage, Papers from the Joint Event CIPA/VAST/EG/EuroMed Event, 2006 